\title{
RADIAL LIMITS OF $M$-SUBHARMONIC FUNCTIONS
}

BY

DAVID ULLRICH

\begin{abstract}
M$-subharmonic" functions are defined in the unit ball of $\mathbf{C}^{n}$. Their basic properties are developed, leading to the following generalization of a theorem of Littlewood: An $M$-subharmonic function such that its restrictions to spheres centered at the origin are bounded in $L^{1}$ must have radial limits almost everywhere on the unit sphere.
\end{abstract}

The purpose of the present paper is to define and develop the basic properties of the class of " $M$-subharmonic" functions in the unit ball of $\mathbf{C}^{n}$ (see Definition 1.15). This is the version of "subharmonic" associated with " $M$-harmonic" (as in [RU]). The paper is divided into four sections:

$\$ 1$ consists of preliminary definitions and a few basic results, concluding with a characterization of those $M$-subharmonic functions which possess "least $M$-harmonic majorants" (Theorems 1.22 and 1.23).

$\$ 2$ proves the Riesz decomposition theorem for $M$-subharmonic functions with a least $M$-harmonic majorant (Theorem 2.16).

$\$ 3$ contains our main theorem: An $M$-subharmonic function satisfying the appropriate growth condition has radial limits almost everywhere (Theorem 3.1). (In the case $n=1$ this was proved by Littlewood [LW].)

$\$ 4$ shows, via a very simple example, that Theorem 3.1 does not extend to admissible limits, nor even to nontangential limits, nor to any sort of approach strictly more general than radial. Note however that an $M$-subharmonic function satisfying stronger growth conditions than those in Theorem 3.1 must have admissible limits a.e. (see [CS]).

This paper is a revised version of the author's thesis written at the University of Wisconsin under the direction of Professor Walter Rudin. It is a pleasure to record here my gratitude for many stimulating discussions on this and various other topics.

1. Preliminaries. Notations are for the most part as in [RU]. Throughout this paper $n$ will be a fixed positive integer, usually $n>1$. (Our results are all standard when $n=1$; including the case $n=1$ would occasionally necessitate dividing our notation, if not our argument, into two cases.) For $z, w \in \mathbf{C}^{n}$ set $\langle z, w\rangle=\sum_{j=1}^{n} z_{j} \bar{w}_{j}$ and $z=\langle z, z\rangle^{1 / 2}$; let $B=\left\{z \in \mathbf{C}^{n}:|z|<1\right\}$ and $S=\left\{\zeta \in \mathbf{C}^{n}:|\zeta|=1\right\}$. For $z \in \mathbf{C}^{n}$ we will sometimes write $z=\left(z_{1}, z^{\prime}\right)$, where $z_{1} \in \mathbf{C}, z^{\prime} \in \mathbf{C}^{n-1}$. For $z, a \in B, a \neq 0$, define

$$
\phi_{a}(z)=\frac{a-P_{a} z-\left(1-|a|^{2}\right)^{1 / 2} Q_{a} z}{1-\langle z, a\rangle},
$$

Received by the editors October 18, 1984.

1980 Mathematics Subject Classification. Primary 32M15; Secondary 31B25. 
where $P_{a} z=\langle z, a\rangle a /\langle a, a\rangle$ is the orthogonal projection of $z$ onto the subspace of $\mathrm{C}^{n}$ spanned by $a$, and $P_{a} z+Q_{a} z=z$. By continuity, let $\phi_{0}(z)=-z$. (Note $\phi_{a}(a)=$ $0, \phi_{a}(0)=a, \phi_{a}\left(\phi_{a}(z)\right)=z$.)

Let $M$ be the group of biholomorphic mappings of $B$ onto $B$. Then $\phi_{a} \in M$ for $a \in B$. Further, any $\psi \in M$ has a unique representation $\psi=U \circ \phi_{a}$ for some $a \in B$ and $U \in \mathscr{U}$; here $\mathscr{U}=\mathscr{U}(n) \subseteq M$ is the unitary group (see, e.g., [RU, Theorems $2.2 .2,2.2 .5])$. It follows that $\psi \in M$ extends to a continuous $\psi: \bar{B} \rightarrow \bar{B} ; \psi(S)=S$.

Let $\sigma$ denote surface area measure on $S$, normalized so that $\sigma(S)=1$. The notations $L^{p}(S),\|f\|_{p}$, etc. refer to $\sigma$.

Suppose $\Omega$ is an open subset of $B, f \in C^{2}(\Omega)$ and $a \in \Omega$; define

$$
\tilde{\Delta} f(a)=\frac{1}{n+1} \Delta\left(f \circ \phi_{a}\right)(0) \text {. }
$$

(Here $\Delta$ is the ordinary Laplacian, obtained by identifying $\mathbf{C}^{n}$ with $\mathbf{R}^{2 n}$.) It follows that

$$
\tilde{\Delta} f(a)=\frac{4}{n+1}\left(1-|a|^{2}\right) \sum_{j, k=1}^{n}\left(\delta_{j, k}-a_{j} \bar{a}_{k}\right) \frac{\partial^{2} f(a)}{\partial z_{j} \partial \bar{z}_{k}},
$$

where as usual $\delta_{j, k}=1$ if $j=k, \delta_{j, k}=0$ if $j \neq k$ (see [RU, Theorem 4.1.3]). The reason for our interest in $\tilde{\Delta}$ is that $\tilde{\Delta}$ is $M$-invariant: if $f \in C^{2}(\Omega)$ and $\psi \in M$ then $\tilde{\Delta}(f \circ \psi)=(\tilde{\Delta} f) \circ \psi$ in $\psi^{-1}(\Omega)$ [RU, Theorem 4.1.2].

1.1 Definition. Suppose $\Omega$ is an open subset of $B, f \in C^{2}(\Omega)$. Then $f$ is M-harmonic in $\Omega$ if $\tilde{\Delta} f=0$ there.

1.2 Definition. If $z \in B$ and $\zeta \in S$, then

$$
P(z, \zeta)=\left\{\frac{1-|z|^{2}}{|1-\langle z, \zeta\rangle|^{2}}\right\}^{n}
$$

If $\mu$ is a (complex Borel) measure on $S$ and $z \in B$, then

$$
P[\mu](z)=\int_{S} P(z, \zeta) d \mu(\zeta)
$$

If $\mu<<\sigma$, that is if $d \mu=f d \sigma, f \in L^{1}(\sigma)$, we will write $P[f]$ in place of $P[\mu]$.

Note that $P[\mu]$ is $M$-harmonic in $B$ for any $\mu$; if $f$ is $M$-harmonic in $B$ and continuous on $\bar{B}$ then $\left.f\right|_{B}=P\left[\left.f\right|_{S}\right.$ ], etc. [RU, §3.3].

The operator $P$ is $M$-invariant:

1.3 Proposition [RU, Theorem 3.3.8]. If $f \in L^{1}(S)$ and $\psi \in M$, then $P[f \circ \psi]=$ $P[f] \circ \psi$.

1.4 TheOREm [RU, Theorem 4.3.3]. Suppose $u$ is M-harmonic in B. There exists a measure $\mu$ such that $u=P[\mu]$ if and only if $\int_{S}|u(r \zeta)| d \sigma(\zeta)$ is a bounded function of $r \in[0,1)$. 
Minor modifications of the proof of Corollary 2 to Theorem 4.2.4 in [RU] suffice to prove the following somewhat stronger theorem:

1.5 TheOREM. Suppose $\Omega$ is an open subset of $B$ and $f$ is measurable and locally bounded in $\Omega$. Then $f$ is M-harmonic in $\Omega$ if and only if

$$
f(a)=\int_{S} f\left(\phi_{a}(r \zeta)\right) d \sigma(\zeta)
$$

for any $a \in \Omega$ and $r>0$ such that $\phi_{a}(r \bar{B}) \subseteq \Omega$.

1.6 Corollary. Suppose $\Omega$ is an open subset of $B, f_{j}$ is $M$-harmonic in $\Omega$ and $f_{j}$ converges to $f$ uniformly on compact subsets of $\Omega$ as $j \rightarrow \infty$. Then $f$ is $M$-harmonic in $\Omega$.

Closely related to Corollary 1.6 is Proposition 1.10 . First a few preliminaries: Let $m$ denote Lebesgue measure in $\mathbf{C}^{n}$.

1.7 Definition. Define a measure $\tau$ in $B$ by $d \tau(z)=\left(1-|z|^{2}\right)^{-n-1} d m(z)$.

The motivation for this definition is that $\tau$ is $M$-invariant: For $f \in L^{1}(\tau)$ and $\psi \in M, \int_{B} f \circ \psi d \tau=\int_{B} f d \tau[\mathbf{R U}$, Theorem 2.2.6].

1.8 Proposition. If $u$ is $M$-harmonic in $\Omega$, an open subset of $B$, and $\phi_{a}(r \bar{B}) \subseteq \Omega$, then

$$
u(a)=\frac{1}{\tau\left(\phi_{a}(r B)\right)} \int_{\phi_{a}(r B)} u d \tau
$$

Proof. By the $M$-invariance of $\tau$ and the fact that $\phi_{a}$ is its own inverse, the right-hand side equals

$$
\frac{1}{\tau(r B)} \int_{r B} u \circ \phi_{a} d \tau
$$

Integrate in polar coordinates and apply Theorem 1.5. Q.E.D.

1.9 LEMMA. If $\Omega$ is a connected open subset of $B, a \in \Omega$ and $K$ is a compact subset of $\Omega$, then there exists $C=C_{K}$ with the following property: If $u \geqslant 0$ is $M$-harmonic in $\Omega$, then $u(z) \leqslant C u(a)$ for all $z \in K$.

Proof. It suffices to consider the case $K=\phi_{a}(r \bar{B}) \subseteq \Omega$. Pick $\varepsilon>0$ such that $\phi_{z}(\varepsilon \bar{B}) \subseteq \Omega$ for all $z \in \phi_{a}(\overline{r B})$; now pick $r_{1}<1$ such that $\phi_{z}(\varepsilon \bar{B}) \subseteq \phi_{a}\left(r_{1} \bar{B}\right) \subseteq \Omega$ for all $z \in \phi_{a}(\overline{r B})$. Then since $u \geqslant 0$, two applications of Proposition 1.8 show that

$$
\begin{aligned}
u(z) & =\frac{1}{\tau\left(\phi_{z}(\varepsilon B)\right)} \int_{\phi_{z}(\varepsilon B)} u d \tau \\
& \leqslant \frac{1}{\tau\left(\phi_{z}(\varepsilon B)\right)} \int_{\phi_{a}\left(r_{1} B\right)} u d \tau=\frac{\tau\left(r_{1} B\right)}{\tau(\varepsilon B)} u(a)
\end{aligned}
$$

for all $z \in \phi_{a}(r \bar{B})$. Q.E.D.

1.10 Proposition. Suppose $\left\{u_{j}\right\}$ is a nondecreasing sequence of M-harmonic functions in the connected open subset $\Omega$ of $B$. Let $u(z)=\lim _{j \rightarrow \infty} u_{j}(z)$. If $u\left(z_{0}\right)<\infty$ for one $z_{0} \in \Omega$, then $u(z)<\infty$ for all $z \in \Omega$ and $u$ is $M$-harmonic in $\Omega$. 
Proof. Replacing $u_{j}$ by $u_{j}-u_{0}$, we may assume $u_{j} \geqslant 0$. Now Lemma 1.9 shows $u$ is locally bounded in $\Omega$, so that Theorem 1.5 and the monotone convergence theorem imply $u$ is $M$-harmonic in $\Omega$. Q.E.D.

Our next goal is to say something about the " $\tilde{\Delta}$-Dirichlet problem" in $r B$ for $0<r<1$ (Theorem 1.14). If $r=1$ this problem is solved by the invariant Poisson kernel $P(z, \zeta)$. Unfortunately $\tilde{\Delta}$ is far from being dilation-invariant [RU, Theorem 4.4.10] so that a solution in $B$ does not automatically lead to a solution in $r B$, as in the Euclidean case. Nevertheless some information can be obtained: We begin with the case of continuous boundary data:

1.11 Proposition [RU, Lemma 5.5.4]. Suppose $f \in C(S), 0<r \leqslant 1$. Then there exists a unique $u \in C(r \bar{B})$ such that $u$ is $M$-harmonic in $r B$ and $u(r \zeta)=f(\zeta)(\zeta \in S)$.

1.12 Definition. For $f \in C(S)$ and $0<r \leqslant 1$ let $P_{r}[f]$ be the M-harmonic function in $r B$ such that $\lim _{\rho \rightarrow r} P_{r}[f](\rho \zeta)=f(\zeta)$ uniformly for $\zeta \in S$. (See Proposition 1.11.)

Note that $P_{r}: C(S) \rightarrow C(r B)$ is linear and positive $\left(P_{r}[f] \geqslant 0\right.$ for $\left.f \geqslant 0\right)$ by the maximum principle [RU, Theorem 4.3.2].

1.13 Lemma. Suppose $0<r_{1}<r_{2} \leqslant 1$. Then there exists $C=C_{r_{1}, r_{2}}$ such that if $f \in C(S)$ and $|z| \leqslant r_{1}$, then $\left|P_{r_{2}}[f](z)\right| \leqslant C\|f\|_{1}$. (Here $\|f\|_{1}=\int_{S}|f| d \sigma$.)

Proof. We may suppose $f$ is real-valued. Let $f^{+}(\zeta)=\max (f(\zeta), 0)$ and $f=f^{+}-f^{-}$; let $u^{+}=P_{r_{2}}\left[f^{+}\right]$and $u^{-}=P_{r_{2}}[f]$. Then Lemma 1.9 shows that for $|z| \leqslant r_{1}$

$$
u^{+}(z) \leqslant C u^{+}(0)=C \int_{S} f^{+} d \sigma
$$

similarly for $u^{-}\left(u^{ \pm} \geqslant 0\right.$ because $\left.f^{ \pm} \geqslant 0\right)$.

Hence linearity of $P_{r_{2}}$ shows

$$
\begin{aligned}
\left|P_{r_{2}}[f](z)\right| & =\left|u^{+}(z)-u^{-}(z)\right| \leqslant u^{+}(z)+u^{-}(z) \leqslant C\left(u^{+}(0)+u^{-}(0)\right) \\
& =C \int_{S}\left(f^{+}+f^{-}\right) d \sigma=C\|f\|_{1} . \quad \text { Q.E.D. }
\end{aligned}
$$

Lemma 1.13 shows that for $0<r<1$ and $z \in r B$ there exists $P_{r, z} \in L^{\infty}(S)$ such that for $f \in C(S), z \in r B$

$$
P_{r}[f](z)=\int_{S} f(\zeta) P_{r, z}(\zeta) d \sigma(\zeta) .
$$

Further, $\left\|P_{r, z}\right\|_{\infty} \leqslant C_{r, r^{\prime}}$ for $|z| \leqslant r^{\prime}<r, P_{r, z} \geqslant 0, \int_{S} P_{r, z} d \sigma=1$. Since $\tilde{\Delta}$ is $\mathscr{U}$ invariant, so is $P_{r, z}: P_{r, U z}=P_{r, z} \circ U^{-1}$ for $U \in \mathscr{U}$. It follows that for $0 \leqslant \rho<r$ we have

$$
\int_{S}\left|P_{r}[f](\rho \zeta)\right| d \sigma(\zeta) \leqslant\|f\|_{1}
$$


Proof. Let $d U$ denote Haar measure on $\mathscr{U}$. Then

$$
\begin{aligned}
& \int_{S}\left|P_{r}[f](\rho \zeta)\right| d \sigma(\zeta)=\int_{\mathscr{U}}\left|P_{r}[f]\left(U \rho e_{1}\right)\right| d U \\
& \leqslant \int_{\mathscr{Q}} \int_{S} P_{r, U_{\rho} e_{1}}(\zeta)|f(\zeta)| d \sigma(\zeta) d U \\
& =\int_{S} \int_{\mathscr{Q}} P_{r, \rho e_{1}}\left(U^{-1} \zeta\right) d U|f(\zeta)| d \sigma(\zeta)=\int_{S}|f(\zeta)| d \sigma(\zeta)
\end{aligned}
$$

by Fubini's theorem and the fact that $\mathscr{U}$ is unimodular (being compact).

Finally, since $P_{r}[f](\rho \zeta) \rightarrow f(\zeta)$ uniformly as $\rho \rightarrow r$ for $f \in C(S)$, the family $\left\{P_{r, \rho e_{1}}: 0<\rho<r\right\}$ must be an approximate identity (for each fixed $r$ ):

Given $\varepsilon>0$, given a neighborhood $A$ of $e_{1}$ in $S$, there exists $\rho_{0}<r$ such that

$$
\int_{S \backslash A} P_{r, \rho e_{1}} d \sigma<\varepsilon \text { for } \rho_{0}<\rho<r .
$$

Using all the various remarks we have made since the proof of Lemma 1.13 one may prove the following theorem by fairly standard arguments:

1.14 THEOREM. Let $0<r \leqslant 1$. For $f \in L^{1}(S)$ there exists a function $P_{r}[f]$ which is $M$-harmonic in $r B$ and which satisfies

$$
\int_{S}\left|P_{r}[f](\rho \zeta)\right| d \sigma(\zeta) \leqslant\|f\|_{1} \quad(0 \leqslant \rho<r)
$$

and

$$
\lim _{\rho \rightarrow r} \int_{S}\left|P_{r}[f](\rho \zeta)-f(\zeta)\right| d \sigma(\zeta)=0
$$

(i.e., $P_{r}[f](\rho \zeta) \rightarrow f(\zeta)$ in $L^{1}(S)$ as $\left.\rho \rightarrow r\right)$.

We come finally to our principal object of study:

1.15 Definition. Suppose $\Omega \subseteq B$ is open and $u: \Omega \rightarrow[-\infty, \infty)$ is upper semicontinuous. Then $u$ is $M$-subharmonic in $\Omega$ if (i) for every $a \in \Omega$ there exists $r(a)>0$ with $\phi_{a}(r(a) \bar{B}) \subseteq \Omega$, such that

$$
u(a) \leqslant \int_{S} u\left(\phi_{a}(r \zeta)\right) d \sigma(\zeta) \quad(0 \leqslant r \leqslant r(a))
$$

and (ii) none of the integrals in (i) are $-\infty$.

NotE. The abbreviations " $M$-sh" and "u.s.c." will be used for " $M$-subharmonic" and "upper semicontinuous".

NoTE. Although we will not be using this fact explicitly, we should point out that, for $u \in C^{2}(\Omega), u$ is $M$-sh if and only if $\tilde{\Delta} u \geqslant 0$.

The following may be proved exactly as in the classical case of subharmonic functions on $\mathbf{R}^{n}$ [HE, p. 59]:

1.16 Proposition. If $u$ is $M$-sh in a neighborhood of the compact set $K \subseteq B$, $v \in C(K)$ is $M$-harmonic in the interior of $K$ and $v \geqslant u$ on the boundary of $K$, then $v \geqslant u$ on all of $K$. 
The class of $M$-sh functions is $M$-invariant:

1.17 Proposition. If $u$ is $M$-sh in the open set $\Omega \subseteq B$ and $\psi \in M$, then $u \circ \psi$ is $M-\operatorname{sh}$ in $\psi^{-1}(\Omega)$.

Proof. Fix $a \in \psi^{-1}(\Omega)$; let $\psi(a)=b \in \Omega$. Now $\phi_{b} \circ \psi \circ \phi_{a} \in M$ and $\phi_{b} \circ \psi \circ \phi_{a}(0)$ $=0$. So a theorem of Cartan (see, e.g., [RU, Theorem 2.1.3]) implies $\phi_{b} \circ \psi \circ \phi_{a}=U$ $\in \mathscr{U}$. Thus, for small enough $r>0$ the unitary invariance of $\sigma$ shows

$$
\begin{aligned}
\int_{S} u \circ \psi\left(\phi_{a}(r \zeta)\right) d \sigma(\zeta) & =\int_{S} u\left(\phi_{b}(U r \zeta)\right) d \sigma(\zeta) \\
& =\int_{S} u\left(\phi_{b}(r \zeta)\right) d \sigma(\zeta) \geqslant u(b)=u \circ \psi(a) . \quad \text { Q.E.D. }
\end{aligned}
$$

1.18 Definition. If $u$ is defined in $B, \zeta \in S$, and $0 \leqslant r<1$, then $u_{r}(\zeta)=u(r \zeta)$.

Note. If $u$ is $M$-sh in $B$, then $u_{r} \in L^{1}(S)$ for $0<r<1: u_{r}$ is bounded above since $u$ is u.s.c., and $\int_{S} u_{r} d \sigma>-\infty$ by (ii) in the definition of " $M$-sh".

1.19 Lemma. If $u$ is $M$-sh in $B$ and $0<r<1$ then $u \leqslant P_{r}\left[u_{r}\right]$ in $r B$. (Here $P_{r}$ is as in Theorem 1.14.)

Proof. Since $u_{r}$ is u.s.c. there exists a sequence $\left\{h_{j}\right\} \subseteq C(S)$ such that $h_{j}(\zeta)$ decreases to $u_{r}(\zeta)$ for all $\zeta \in S$. Now Proposition 1.16 shows that $P_{r}\left[h_{j}\right] \geqslant u$ in $r B$; the monotone convergence theorem then implies that $P_{r}\left[u_{r}\right] \geqslant u$ in $r B$.

1.20 Corollary. If $u$ is $M$-sh in $B$ and $0<r_{1}<r_{2}<1$, then $P_{r_{1}}\left[u_{r_{1}}\right] \leqslant P_{r_{2}}\left[u_{r_{2}}\right]$ in $r_{1} B$.

Proof. Let $v=P_{r_{2}}\left[u_{r_{2}}\right]$. Then $v=P_{r_{1}}\left[v_{r_{1}}\right]$ in $r_{1} B$ and Lemma 1.19 implies $v_{r_{1}} \geqslant u_{r_{1}}$. Hence

$$
P_{r_{2}}\left[u_{r_{2}}\right]=P_{r_{1}}\left[v_{r_{1}}\right] \geqslant P_{r_{1}}\left[u_{r_{1}}\right]
$$

in $r_{1} B$. Q.E.D.

Note. By unitary invariance of $P_{r}, P_{r}\left[u_{r}\right](0)=\int_{S} u(r \zeta) d \sigma(\zeta)$. Hence Corollary 1.20 contains the statement that

$$
\int_{S} u\left(r_{1} \zeta\right) d \sigma(\zeta) \leqslant \int_{S} u\left(r_{2} \zeta\right) d \sigma(\zeta) \text { for } r_{1}<r_{2}, u M \text {-sh. }
$$

1.21 Definition. Suppose $u$ is defined in B. Then $v$ is an M-harmonic majorant of $u$ if $v$ is $M$-harmonic and $v \geqslant u$. If $v_{0}$ is an M-harmonic majorant of $u$ and $v_{0} \leqslant v$ for all $M$-harmonic majorants $v$, then $v_{0}$ is the least $M$-harmonic majorant of $u$.

We finally state

1.22 THEOREM. If $u$ is $M$-sh in $B$, then the following are equivalent:

(i) $u$ has a least $M$-harmonic majorant.

(ii) $u$ has an M-harmonic majorant.

(iii) $\int_{S} u(r \zeta) d \sigma(\zeta)$ is bounded above for $0 \leqslant r<1$.

Proof. It is trivial that (i) implies (ii) and that (ii) implies (iii).

Suppose (iii). Then (i) follows by a standard construction: Pick a sequence $r_{j}$ increasing to 1 . Define $v_{j}$ in $r_{j} B$ by $v_{j}=P_{r_{j}}\left[u_{r_{j}}\right]$. Given $r<1, v_{j}$ is defined in $r B$ for all but finitely many values of $j$; Corollary 1.20 shows that $\left\{v_{j}\right\}$ increases to some 
limit, say $v$. Since

$$
v_{j}(0)=P_{r_{j}}\left[u_{r_{j}}\right](0)=\int_{S} u\left(r_{j} \zeta\right) d \sigma(\zeta)
$$

(iii) shows that $v(0)<\infty$; now Proposition 1.10 shows $v$ is $M$-harmonic; Lemma 1.19 and the positivity of $P_{r}$ show that $v$ is the least $M$-harmonic majorant of $u$. Q.E.D.

Similarly:

1.23 THEOREM. If $u$ is $M$-sh in $B$, then the following are equivalent:

(i) $u$ has a least $M$-harmonic majorant of the form $P[\mu]$ for some (Borel) measure $\mu$ on $S$.

(ii) For some $\mu, P[\mu] \geqslant u$.

(iii) $\int_{S} u^{+}(r \zeta) d \sigma(\zeta)$ is bounded for $0 \leqslant r<1$.

(iv) $\int_{S}|u(r \zeta)| d \sigma(\zeta)$ is bounded for $\frac{1}{2} \leqslant r<1$. (Note: If $u(0)=-\infty$, the integral in (iv) cannot be bounded for $0<r<1$.)

Proof. As before (i) trivially implies (ii), and (ii) trivially implies (iii); the other two implications are easy:

Suppose (iii). Since $u^{+}$is $M$-sh, $\int_{S} u^{+}(r \zeta) d \sigma(\zeta)$ is an increasing function of $r$ (see the Note following Corollary 1.20) so $\lim _{r \rightarrow 1} \int_{S} u^{+}(r \zeta) d \sigma(\zeta)$ exists. Since $u \leqslant u^{+}$and $u$ is $M$-sh, $\lim _{r \rightarrow 1} \int_{S} u(r \zeta) d \sigma(\zeta)$ exists. But $|u|=2 u^{+}-u$, so $\lim _{r \rightarrow 1} \int_{S}|u(r \zeta)| d \sigma(\zeta)$ exists; this gives (iv).

Now suppose (iv). Since $u \leqslant|u|$, Theorem 1.22 implies that $u$ has a least $M$ harmonic majorant $v$. The construction of $v$ in the proof of Theorem 1.22, together with Theorem 1.14, implies that for $r<1$

$$
\begin{aligned}
\int_{S}|v(r \zeta)| d \sigma(\zeta) & =\lim _{j \rightarrow \infty} \int_{S}\left|v_{j}(r \zeta)\right| d \sigma(\zeta) \\
& =\lim _{j \rightarrow \infty} \int_{S}\left|P_{r_{j}}\left[u_{r_{j}}\right](r \zeta)\right| d \sigma(\zeta) \\
& \leqslant \lim _{j \rightarrow \infty} \int_{S}\left|u_{r_{j}}\right| d \sigma \leqslant \sup _{1 / 2 \leqslant \rho<1} \int_{S}|u(\rho \zeta)| d \sigma(\zeta)
\end{aligned}
$$

so that Theorem 1.4 implies $v=P[\mu]$, giving (i). Q.E.D.

2. The Riesz decomposition. Recall that $\tau$ is an $M$-invariant measure in $B$ (Definition 1.7).

2.1 Definition. For any functions $f, h$ on $B$ such that the integral is defined,

$$
f * h(z)=\int_{B} f\left(h \circ \phi_{z}\right) d \tau .
$$

The invariance of $\tau$ and the fact that $\phi_{z}^{-1}=\phi_{z}$ imply that $f * h=h * f$. If $f$, $h \in L^{1}(\tau)$ and one of $f$ and $h$ is radial, then Fubini's theorem and the fact that $\left|\phi_{z}(w)\right|=\left|\phi_{w}(z)\right|$ show that $f * h$ is defined almost everywhere and $\int_{B} f * h d \tau=$ $\int_{B} f d \tau \int_{B} h d \tau$. 
Note. In general $\int_{B} f * h d \tau \neq \int_{B} f d \tau \int_{B} h d \tau$. (For example, in case $n=1$ : if $\int_{B} f d \tau=\int_{B} h d \tau=1, f, h \geqslant 0, f$ is concentrated near $a \in B$ and $h$ is concentrated near $-a$, one may calculate that $\int_{B} f * h d \tau$ is approximately $\left(1-|a|^{2}\right)\left(1+|a|^{2}\right)^{-1}$.) This problem could be avoided by taking $\int_{B} f(w) h\left(\phi_{w}(z)\right) d \tau(w)$ as the definition of $f * h(z)$, but this approach leads to other problems. We are interested in this convolution simply as a tool: In each of the applications below one of the functions is radial, so this problem does not arise.

2.2 Proposition. If $f, \chi, h \in L^{1}(\tau)$ and $\chi$ is radial, then $(f * \chi) * h=f *(\chi * h)$.

Proof. Fix $a, w \in B$. Note that $\phi_{\phi_{a}(w)} \circ \phi_{a} \circ \phi_{w}(0)=0$, so that $\phi_{\phi_{a}(w)} \circ \phi_{a} \circ \phi_{w}=U$ $\in \mathscr{U}\left[\mathbf{R U}\right.$, Theorem 2.1.3] which implies $\phi_{\phi_{a}(w)}=U \circ \phi_{w} \circ \phi_{a}$. Hence, since $\tau$ is $M$-invariant, $\left|U \phi_{w}(z)\right|=\left|\phi_{z}(w)\right|$ and $\chi$ is radial,

$$
\begin{aligned}
\int_{B} \chi(z) h\left(\phi_{\phi_{a}(w)}(z)\right) d \tau(z) & =\int_{B} \chi\left(U \phi_{w}\left(\phi_{a}(z)\right)\right) h(z) d \tau(z) \\
& =\int_{B} \chi\left(U \phi_{w}(z)\right) h\left(\phi_{a}(z)\right) d \tau(z) \\
& =\int_{B} \chi\left(\phi_{z}(w)\right) h\left(\phi_{a}(z)\right) d \tau(z)
\end{aligned}
$$

Thus

$$
\begin{aligned}
(f * \chi) * h(a) & =\int_{B} \int_{B} f(w) \chi\left(\phi_{z}(w)\right) h\left(\phi_{a}(z)\right) d \tau(w) d \tau(z) \\
& =\int_{B} \int_{B} f(w) \chi(z) h\left(\phi_{\phi_{a}(w)}(z)\right) d \tau(z) d \tau(w) \\
& =f *(\chi * h)(a) \quad \text { Q.E.D. }
\end{aligned}
$$

The following lemma may be proved by imitating the proof of Theorem 4.1.3(i) of [RU]:

2.3 LemMa. For $0<r<1$ let

$$
\chi_{r}(z)= \begin{cases}\omega_{n, r}, & |z| \leqslant r, \\ 0, & |z|>r,\end{cases}
$$

where $\omega_{n, r}$ is chosen so that $\int_{B} \chi_{r} d \tau=1$. (Thus $\omega_{n, r} \sim c r^{-2 n}$ as $r \rightarrow 0$.) There exists $a$ constant $C_{n}$ such that for $f \in C_{c}^{2}(B)$

$$
\tilde{\Delta} f=\lim _{r \rightarrow 0} \frac{C_{n}}{r^{2}}\left(f * \chi_{r}-f\right)
$$

uniformly on $B$.

2.4 Proposition. For $u, v \in C_{c}^{2}(B)$

$$
\int_{B} u \tilde{\Delta} v d \tau=\int_{B} v \tilde{\Delta} u d \tau
$$


Proof. Let $u_{1}(z)=u(-z)$ and let $\chi_{r}$ be as in Lemma 2.3. Since $\chi_{r}$ is radial, Proposition 2.2 shows that

$$
\begin{aligned}
\int_{B} u \tilde{\Delta} v d \tau & =\left(u_{1} * \tilde{\Delta} v\right)(0) \\
& =\lim _{r \rightarrow 0} \frac{c_{n}}{r^{2}} u_{1} *\left(v * \chi_{r}-v\right)(0) \\
& =\lim _{r \rightarrow 0} \frac{c_{n}}{r^{2}}\left(u_{1} * \chi_{r}-u_{1}\right) * v(0) \\
& =\left(\tilde{\Delta} u_{1}\right) * v(0)=\int_{B}(\tilde{\Delta} u) v d \tau . \quad \text { Q.E.D. }
\end{aligned}
$$

2.5 TheOREM. Suppose $u$ is $M$-sh in B. There exists a (Borel) measure $\mu \geqslant 0$ in $B$ such that $\int_{B} u \tilde{\Delta} v d \tau=\int_{B} v d \mu$ for $v \in C_{c}^{2}(B)$ (i.e., " $d \mu=\tilde{\Delta} u d \tau$ " in the sense of distributions; see Proposition 2.4).

Proof. We need only show that if $v \in C_{c}^{2}(B)$ and $v \geqslant 0$, then $\int_{B} u \tilde{\Delta} v d \tau \geqslant 0$. Let $\chi_{r}$ be as in Lemma 2.3; let $u_{1}(z)=u(-z)$. There exists a compact $K \subseteq B$ such that for $0<r \leqslant \frac{1}{2}$ the function $\chi_{r} * v$ is supported on $K$. Since $u$ is locally integrable (see remarks preceding Lemma 1.19) and the convergence in Lemma 2.3 is uniform, we see that

$$
\begin{aligned}
\int_{B} u \tilde{\Delta} v d \tau & =u_{1} * \tilde{\Delta} v(0)=\lim _{r \rightarrow 0} \frac{c_{n}}{r^{2}} u_{1} *\left(v * \chi_{r}-v\right)(0) \\
& =\lim _{r \rightarrow 0} \frac{c_{n}}{r^{2}}\left(u_{1} * \chi_{r}-u_{1}\right) * v(0) \geqslant 0 .
\end{aligned}
$$

(Since $u$ is $M$-sh, so is $u_{1}$; now the definition of " $M$-sh" and an integration in polar coordinates show $u_{1} * \chi_{r} \geqslant u_{1}$.) Q.E.D.

We now introduce the "Green's function" associated with $\tilde{\Delta}$ :

2.6 Definition. For $z \in B$

$$
g(z)=c_{n} \int_{|z|}^{1}\left(1-t^{2}\right)^{n-1} t^{-2 n+1} d t .
$$

(Here $c_{n}$ is a positive constant, the value of which will be determined in the proof of Proposition 2.8.)

NoTE. $g: B \rightarrow(0, \infty] ; g(0)=\infty$.

2.7 Lemma. The function $-g$ is $M$-sh in $B$.

Proof. One sees that $g$ is $C^{2}$ in $B \backslash\{0\}$ and that $\tilde{\Delta} g(z)=0$ for $z \neq 0$. Hence if $z \neq 0$ and $0<r<|z|=r(z)$,

$$
-g(z)=\int_{S}(-g)\left(\phi_{z}(r \zeta)\right) d \sigma(\zeta)
$$

On the other hand, $-g(0)=-\infty$, so for any $r>0$

$$
-g(0) \leqslant \int_{S}(-g)\left(\phi_{0}(r \zeta)\right) d \sigma(\zeta) \quad \text { Q.E.D. }
$$


2.8 Proposition. If the constant $c_{n}>0$ is properly chosen in Definition 2.6, then $-\int_{B} g \tilde{\Delta} v d \tau=v(0)$ for all $v \in C_{c}^{2}(B)$.

Proof. Let $\mu \geqslant 0$ be the measure such that " $\tilde{\Delta}(-g) d \tau=d \mu$ " in the sense of Theorem 2.5 ( $-g$ is $M$-sh by Lemma 2.7). Since $g$ is $M$-harmonic in $B \backslash\{0\}$, it follows from Proposition 2.4 that support $(\mu) \subseteq\{0\}$. On the other hand, $\mu \neq 0$ since $g$ is certainly not $M$-harmonic in $B$. So if $c_{n}$ is properly chosen in Definition 2.6 , then

$$
\int_{B} v d \mu=v(0) \quad\left(v \in C_{c}^{2}(B)\right) \text { Q.E.D. }
$$

2.9 Corollary. For $v \in C_{c}^{2}(B),-g * \tilde{\Delta} v=v$.

Proof. Fix $z \in B$; let $u=v \circ \phi_{z}$. Then

$$
-g * \tilde{\Delta} v(z)=-\int_{B} g(\tilde{\Delta} v) \circ \phi_{z} d \tau=-\int_{B} g \tilde{\Delta} u d \tau=u(0)=v(z)
$$

by the $M$-invariance of $\tilde{\Delta}$. Q.E.D.

2.10 Lemma. If $h \in C_{c}^{2}(B)$ and $v=-h * g$, then $\tilde{\Delta} v=h$.

Proof. Let $u \in C_{c}^{2}(B)$ and $u_{1}(z)=u(-z)$. Then

$$
\begin{aligned}
\int_{B} u \tilde{\Delta} v d \tau & =\int_{B}(\tilde{\Delta} u) v d \tau \\
& =\left(\left(\tilde{\Delta} u_{1}\right) * v\right)(0)=-\left(\tilde{\Delta} u_{1}\right) *(g * h)(0) \\
& =-\left(\tilde{\Delta} u_{1} * g\right) * h(0)=u_{1} * h(0)=\int_{B} u h d \tau .
\end{aligned}
$$

Since this holds for any $u \in C_{c}^{2}(B), \tilde{\Delta} v=h$. Q.E.D.

2.11 Definition. If $f$ is a function on $B$ such that the integral is defined, then

$$
\begin{aligned}
f^{\#}(z) & =\int_{S} f(|z| \zeta) d \sigma(\zeta)=\int_{\mathscr{U}} f(U z) d U \\
& =\text { the radialization of } f \text { at } z .
\end{aligned}
$$

2.12 Lemma. Suppose $\chi \in C_{c}^{2}(B), \chi$ is radial and $\int \chi d \tau=0$. Let $v=-g * \chi$. Then $\tilde{\Delta} v=\chi$ and $v$ has compact support.

Proof. Lemma 2.10 shows $\tilde{\Delta} v=\chi$; we need only show $v$ has compact support.

Note that if $|z|>|w|$, then $g \circ \phi_{z}$ is $M$-harmonic in a neighborhood of $|w| \bar{B}$, so that $\left(g \circ \phi_{z}\right)^{\#}(w)=g \circ \phi_{z}(0)=g(z)$.

Pick $r<1$ such that $\chi$ has support in $r \bar{B}$. Since $\chi$ is radial it follows that for $|z|>r$

$$
\begin{aligned}
g * \chi(z) & =\int_{B}\left(g \circ \phi_{z}\right)(w) \chi(w) d \tau(w) \\
& =\int_{B} \int_{\mathscr{U}}\left(g \circ \phi_{z}\right)(U w) \chi(w) d U d \tau(w) \\
& =\int_{B}\left(g \circ \phi_{z}\right)^{\#}(w) \chi(w) d \tau(w) \\
& =g(z) \int_{B} \chi d \tau=0 . \quad \text { Q.E.D. }
\end{aligned}
$$


2.13 Lemma. Suppose $\left\{r_{j}\right\}$ decreases to zero as $j \rightarrow \infty$; suppose $X_{j} \geqslant 0, X_{j}$ is radial, $\int_{B} \chi_{j} d \tau=1$, and $\operatorname{support}\left(\chi_{j}\right) \subseteq\left\{z: r_{j+1} \leqslant|z| \leqslant r_{j}\right\}$. Then for any $u M$-sh in $B$ and any $z \in B$ the sequence $u * \chi_{j}(z)$ decreases to $u(z)$ as $j \rightarrow \infty$,

Proof. Fix $z \in B$. Suppose $t>u(z)$. Since $u$ is u.s.c. there exists $r>0$ such that $u(w)<t$ for $w \in \phi_{z}(r B)$. Hence for $j$ large enough that $r_{j}<r$

$$
u * \chi_{j}(z)=\int_{B} u(w) \chi_{j}\left(\phi_{z}(w)\right) d \tau(w) \leqslant t \int_{B} \chi_{j} d \tau=t,
$$

i.e., $\lim \sup _{j \rightarrow \infty} u * \chi_{j}(z) \leqslant u(z)$. On the other hand, integration in polar coordinates shows $u * \chi_{j} \geqslant u$; hence $\lim _{j \rightarrow \infty} u * \chi_{j}(z)=u(z)$. Similarly the remark following Corollary 1.20 shows that $u * \chi_{j}$ decreases as $j \rightarrow \infty$. Q.E.D.

2.14 TheOREM. Suppose $u \leqslant 0$ is $M$-sh in $B$ and $\lim _{r \rightarrow 1} \int_{s} u(r \zeta) d \sigma(\zeta)=0$. Then $u(0)=-\int_{B} g d \mu$, where " $d \mu=\tilde{\Delta} u d \tau$ " in the sense of Theorem 2.5 .

Proof. Pick some sequence $r_{j}$ decreasing to zero. Let

$$
A_{j}^{1}=\left\{z: r_{j+1} \leqslant|z| \leqslant r_{j}\right\} \text { and } A_{j}^{2}=\left\{z: 1-r_{j} \leqslant|z| \leqslant 1-r_{j+1}\right\} .
$$

For $j=1,2,3, \ldots$ and $k=1,2$ let $\chi_{j}^{k} \geqslant 0$ be a radial $C_{c}^{2}$ function with support contained in $A_{j}^{k}$ such that $\int_{B} \chi_{j}^{k} d \tau=1$. Let $\chi_{j}=\chi_{j}^{1}-\chi_{j}^{2}$. Lemma 2.13 shows that

$$
u(0)=\lim _{j \rightarrow \infty} \int_{B} u \chi_{j}^{1} d \tau
$$

our hypothesis on $u$ and the fact that $\chi_{j}^{2}$ is radial and positive show that $\lim _{j \rightarrow \infty} \int_{B} u \chi_{j}^{2} d \tau=0$. Hence $u(0)=\lim _{j \rightarrow \infty} \int_{B} u \chi_{j} d \tau$.

Since $-g$ is $M$-sh, Lemma 2.13 shows that $g * \chi_{j}^{1}$ increases to $g$ at every point. The same proof shows $g * \chi_{j}^{2}$ decreases to zero pointwise, so $g * \chi_{j}$ increases to $g$ pointwise. Let $v_{j}=-g * \chi_{j}$, so $\tilde{\Delta} v_{j}=\chi_{j}, v_{j} \in C_{c}^{2}(B)$ (Lemma 2.12; note $\int_{B} \chi_{j} d \tau=$ 0 ). The monotone convergence theorem shows

$$
\begin{aligned}
u(0) & =\lim _{j \rightarrow \infty} \int_{B} u \chi_{j} d \tau=\lim _{j \rightarrow \infty} \int_{B} u \tilde{\Delta} v_{j} d \tau \\
& =\lim _{j \rightarrow \infty} \int_{B} v_{j} d \mu=\lim _{j \rightarrow \infty}-\int_{B}\left(g * \chi_{j}\right) d \mu=-\int_{B} g d \mu . \quad \text { Q.E.D. }
\end{aligned}
$$

2.15 Definition. For $z, w \in B, G(z, w)=g\left(\phi_{z}(w)\right)$.

We can finally prove the Riesz Decomposition Theorem:

2.16 THEOREM. Suppose $u$ is $M$-sh in $B$ and has an M-harmonic majorant. Let $h$ be the least M-harmonic majorant of $u$, and let $\mu$ be the measure in $B$ with " $d \mu=\tilde{\Delta} u d \tau$ " (Theorem 2.5). Then for all $z \in B$

$$
u(z)=h(z)-\int_{B} G(z, w) d \mu(w) .
$$

Proof. Fix $z \in B$. Let $u_{1}=u \circ \phi_{z}-h \circ \phi_{z}$. Then $u_{1}$ is $M$-sh and has least $M$-harmonic majorant 0 ; hence the proof of Theorem 1.22 shows

$$
\lim _{r \rightarrow 1} \int_{S} u_{1}(r \zeta) d \sigma(\zeta)=0 .
$$


So, by Theorem 2.14, $u_{1}(0)=-\int_{B} g d \mu_{1}$, where " $d \mu_{1}=\tilde{\Delta} u_{1} d \tau$ ". Since $h$ is $M$ harmonic, Proposition 2.4 shows that

$$
\begin{aligned}
\int v d \mu_{1} & =\int_{B} u_{1} \tilde{\Delta} v d \tau \\
& =\int_{B}\left(u \circ \phi_{z}\right) \tilde{\Delta} v d \tau-\int_{B}\left(h \circ \phi_{z}\right) \tilde{\Delta} v d \tau \\
& =\int_{B} u \tilde{\Delta}\left(v \circ \phi_{z}\right) d \tau-\int_{B} \tilde{\Delta}\left(h \circ \phi_{z}\right) v d \tau \\
& =\int_{B}\left(v \circ \phi_{z}\right) d \mu-0
\end{aligned}
$$

for any $v \in C_{c}^{2}(B)$, hence for any Borel measurable $v \geqslant 0$. Thus

$$
u(z)-h(z)=u_{1}(0)=-\int_{B} g d \mu_{1}=\int_{B} g \circ \phi_{z} d \mu . \quad \text { Q.E.D. }
$$

2.17 CoRollaRy. Under the hypothesis of Theorem $2.16 \mathrm{~g} \circ \phi_{z} \in L^{1}(\mu)$ for almost every $z \in B$. ("Almost every" refers to Lebesgue measure.)

Proof. By clause (ii) in Definition $1.15 u(z)>-\infty$ almost everywhere. Q.E.D.

3. Radial limits. Our main result is

3.1 Theorem. Suppose $u$ is $M$-sh in B and

$$
\sup _{1 / 2 \leqslant r<1} \int_{S}|u(r \zeta)| d \sigma(\zeta)<\infty .
$$

Then $\lim _{r \rightarrow 1} u(r \zeta)$ exists for almost all $\zeta \in S$.

By the remark following Corollary 1.20, this immediately implies:

3.2 Corollary. Suppose $u \leqslant 0$ is $M$-sh in B. Then $\lim _{r \rightarrow 1} u(r \zeta)$ exists for almost all $\zeta \in S$.

THEOREM 3.1 will follow from the following theorem:

3.3 Theorem. Suppose $\mu \geqslant 0$ is a (Borel) measure in $B$ with $\int_{B} g d \mu<\infty$. Then $v(z)=\int_{B} G(z, w) d \mu(w)<\infty$ for almost all $z \in B$, and $\lim _{r \rightarrow 1} v(r \zeta)=0$ for almost all $\zeta \in S$.

Proof that Theorem 3.3 implies Theorem 3.1. Let $u$ be as in Theorem 3.1. By Theorem 1.23 there exists a measure $\nu$ on $S$ such that $h=P[\nu]$ is the least $M$-harmonic majorant of $u$; Theorem 2.16 implies that for all $z \in B$

$$
u(z)=h(z)-\int_{B} G(z, w) d \mu(w),
$$

where " $d \mu=\tilde{\Delta} u d \tau$ ". A theorem of Koranyi [RU, Theorem 5.49] shows that $h$ has admissible limits almost everywhere. Let $v(z)=\int_{B} G(z, w) d \mu(w)$; we are done if we show $v(r \zeta) \rightarrow 0$ as $r \rightarrow 1$ for almost every $\zeta \in S$. If $u(0)=-\infty$, then $\int_{B} g d \mu=\infty$, so Theorem 3.3 does not quite apply directly. However: Let $\mu=\mu_{1}+\mu_{2}$, where $\mu_{1}$ has compact support and $\mu_{2}$ vanishes in some neighborhood of $0\left(\mu_{j} \geqslant 0\right)$. Pick $a \in B$ 
with $u(a)>-\infty$; thus $\int_{B} g \circ \phi_{a} d \mu<\infty$. Now $g(z)\left(g\left(\phi_{a}(z)\right)\right)^{-1}$ is bounded if $z$ stays away from zero; hence $\int_{B} g d \mu_{2}<\infty$. Let $v_{j}(z)=\int_{B} G(z, w) d \mu_{j}(w)$. Theorem 3.3 shows $v_{2}(r \zeta) \rightarrow 0$ a.e. But $\mu_{1}$ has compact support so $v_{1}(r \zeta) \rightarrow 0$ uniformly on $S$ as $r \rightarrow 1$; hence $v(r \zeta) \rightarrow 0$ a.e. Q.E.D.

The rest of this section will be devoted to the proof of Theorem 3.3. Throughout this section we will assume that $\mu$ is a positive measure in $B$ with $\int_{B} g d \mu<\infty$. Since, as above, $\int_{B} G(z, w) d \mu(w)$ approaches zero uniformly as $|z| \rightarrow 1$ if $\mu$ has compact support, we may (and shall!) assume $\mu\left(\frac{1}{2} B\right)=0$.

3.4 Definition. For $z \in B$,

$$
g_{0}(z)=\left\{\begin{array}{l}
g(z), \quad|z|<\frac{1}{2}, \\
0, \quad|z| \geqslant \frac{1}{2},
\end{array}\right.
$$

$g_{1}=g-g_{0}$. Let $G_{0}(z, w)=g_{0}\left(\phi_{z}(w)\right), G_{1}=G-G_{0}$.

3.5 Lemma. For $z, w \in B(w \neq 0)$

$$
\frac{g_{1}\left(\phi_{z}(w)\right)}{g(w)} \leqslant C \frac{(1-|z|)^{n}}{|1-\langle z, w /|w|\rangle|^{2 n}} .
$$

Proof. If $\left|\phi_{z}(w)\right|<\frac{1}{2}$, then $g_{1}\left(\phi_{z}(w)\right)=0$. Suppose $\left|\phi_{z}(w)\right| \geqslant \frac{1}{2}$. Now

$$
|g(a)| \geqslant C\left(1-|a|^{2}\right)^{n} \quad \text { for all } a \in B,
$$

and

$$
|g(a)| \leqslant C\left(1-|a|^{2}\right)^{n} \text { for }|a| \geqslant \frac{1}{2}
$$

hence

$$
\begin{aligned}
\frac{g_{1}\left(\phi_{z}(w)\right)}{g(w)} & \leqslant C\left\{\frac{1-\left|\phi_{z}(w)\right|^{2}}{1-|w|^{2}}\right\}^{n}=C \frac{\left(1-|z|^{2}\right)^{n}}{|1-\langle z, w\rangle|^{2 n}} \\
& \leqslant C \frac{\left(1-|z|^{2}\right)^{n}}{|1-\langle z, w /|w|\rangle|^{2 n}} .
\end{aligned}
$$

Here we have used the facts that

$$
1-\left|\phi_{z}(w)\right|^{2}=\frac{\left(1-|z|^{2}\right)\left(1-|w|^{2}\right)}{|1-\langle z, w\rangle|^{2}}
$$

[RU, Theorem 2.2.2] and that $|1-\langle z, w\rangle| \geqslant \frac{1}{2}|1-\langle z, w /|w|\rangle|$. Q.E.D.

3.6 Definition. Define a measure $\tilde{\mu}$ on $S$ by

$$
\int_{S} \chi d \tilde{\mu}=\int_{B} \chi\left(\frac{z}{|z|}\right) g(z) d \mu(z) .
$$

(This is well defined because of our standing assumptions $\int_{B} g d \mu<\infty$ and $\mu\left(\frac{1}{2} B\right)=$ 0.)

3.7 Proposition. For $z \in B$

$$
\int_{B} G_{1}(z, w) d \mu(z) \leqslant c P[\tilde{\mu}](z) .
$$


Proof. By Lemma 3.5

$$
\begin{aligned}
\int_{B} G_{1}(z, w) d \mu(w) & =\int_{B} \frac{g_{1}^{\circ} \phi_{z}}{g} g d \mu \\
& \leqslant c \int_{B} \frac{\left(1-|z|^{2}\right)^{n}}{|1-\langle z, w /|w|\rangle|^{2 n}} g(w) d \mu(w) \\
& =c \int_{s} \frac{\left(1-|z|^{2}\right)^{n}}{|1-\langle z, \zeta\rangle|^{2 n}} d \tilde{\mu}(\zeta)=c P[\tilde{\mu}](z) . \quad \text { Q.E.D. }
\end{aligned}
$$

3.8 Corollary. For $\lambda>0$

$$
\sigma\left(\left\{\zeta \in S: \sup _{0 \leqslant r<1} \int_{B} G_{1}(r \zeta, w) d \mu(w)>\lambda\right\}\right) \leqslant \frac{c}{\lambda} \int_{B} g d \mu .
$$

Proof. Combining Theorems 5.2.4 and 5.4.5 of [RU] shows that

$$
\sigma\left(\left\{\zeta \in S: \sup _{0 \leqslant r<1} P[\tilde{\mu}](r \zeta)>\lambda\right\}\right) \leqslant \frac{c}{\lambda}\|\tilde{\mu}\| .
$$

(In fact, the theorems in [RU] give a much stronger result!) So, noting that $\|\tilde{\mu}\|=\int_{B} g d \mu$, the desired inequality follows directly from Proposition 3.7. Q.E.D.

We will be done when we have established the analogue of Corollary 3.8 with " $G_{1}$ " replaced by " $G_{0}$ " (Proposition 3.19). This requires a bit of preparation:

3.9 Definition. For $z \in B, 0<r<1$, set $V_{r}(z)=\phi_{z}(r B)$. For $\zeta \in S, 0<R<1$, $0<r<1$, set $Q_{R}^{r}(\zeta)=\pi\left(V_{r}(R \zeta)\right)$, where $\pi: B \backslash\{0\} \rightarrow S$ is defined by $\pi(z)=z /|z|$.

3.10 Lemma. For $z, a, w \in B,\left|\phi_{z}\left(\phi_{a}(w)\right)\right|=\left|\phi_{\phi_{a}(z)}(w)\right|$.

Proof. As in the proof of Proposition 2.2, there exists $U \in \mathscr{U}$ with $\phi_{z} \circ \phi_{a}=$ $U \circ \phi_{\phi_{u}(z)}$. Q.E.D.

3.11 LEMMA. $\phi_{a}\left(V_{r}(z)\right)=V_{r}\left(\phi_{a}(z)\right)$.

Proof. By Lemma 3.10 the following are equivalent:

$$
\begin{gathered}
w \in \phi_{a}\left(V_{r}(z)\right) \\
\phi_{a}(w) \in V_{r}(z) \\
\phi_{z}\left(\phi_{a}(w)\right) \in r B \\
\phi_{\phi_{a}(z)}(w) \in r B \\
w \in V_{r}\left(\phi_{a}(z)\right) .
\end{gathered}
$$

Q.E.D.

3.12 LemMA. For $a, z \in B,\left|\phi_{a}(z)\right| \leqslant|a|+|z|$.

Proof. One checks that for $x, y \in[0,1]$

$$
1-(x+y)^{2} \leqslant \frac{\left(1-x^{2}\right)\left(1-y^{2}\right)}{(1+x y)^{2}} .
$$


It follows that

$$
\begin{aligned}
1-(|a|+|z|)^{2} & \leqslant \frac{\left(1-|a|^{2}\right)\left(1-|z|^{2}\right)}{(1+|a||z|)^{2}} \\
& \leqslant \frac{\left(1-|a|^{2}\right)\left(1-|z|^{2}\right)}{|1-\langle z, a\rangle|^{2}}=1-\left|\phi_{a}(z)\right|^{2}
\end{aligned}
$$

[RU, Theorem 2.2.2]. Q.E.D.

The following is a key step in the proof of the "covering lemma" needed for Lemma 3.16:

3.13 LEMMA. If $V_{r}(z) \cap V_{r^{\prime}}\left(z^{\prime}\right) \neq \varnothing$ and $r^{\prime} \leqslant r$, then $V_{r^{\prime}}\left(z^{\prime}\right) \subseteq V_{3 r}(z)$. (For $r \geqslant 1$ set $V_{r}(z)=B$.)

Proof. By Lemma 3.11 we may assume $z=0: r B \cap V_{r^{\prime}}\left(z^{\prime}\right) \neq \varnothing$; i.e, there exists $w$ with $|w|<r,\left|\phi_{z^{\prime}}(w)\right|<r^{\prime} \leqslant r$. Now Lemma 3.12 shows

$$
\left|z^{\prime}\right|=\left|\phi_{w}\left(\phi_{w}\left(z^{\prime}\right)\right)\right| \leqslant|w|+\left|\phi_{w}\left(z^{\prime}\right)\right| \leqslant 2 r .
$$

So for any $y \in V_{r^{\prime}}\left(z^{\prime}\right)$

$$
|y|=\left|\phi_{z^{\prime}}\left(\phi_{z^{\prime}}(y)\right)\right| \leqslant\left|z^{\prime}\right|+\left|\phi_{z^{\prime}}(y)\right| \leqslant 2 r+r^{\prime} \leqslant 3 r,
$$

i.e., $V_{r^{\prime}}\left(z^{\prime}\right) \subseteq 3 r B$. Q.E.D.

3.14 LEMMA. For $0<r \leqslant \frac{1}{2}, \frac{1}{2}<R<1, \zeta \in S$, $\sigma\left(Q_{R}^{r}(\zeta)\right) \sim\left(1-R^{2}\right)^{n} r^{2 n-1}$. ( Here $A \sim B$ means $A B^{-1}$ and $A^{-1} B$ are bounded.)

Proof. By unitary invariance, we may assume $\zeta=e_{1}$. For $r, \rho$ small, let

$$
\Omega_{\rho}^{r}=\left\{t e^{i \theta}: 0<1-t<\rho^{2} r^{2},|\theta|<\rho^{2} r\right\},
$$

and set $N_{\rho}^{r}=\left\{\zeta \in S: \zeta_{1} \in \Omega_{\rho}^{r}\right\}$. Formula 1.4.5(2) in [RU] and integration in polar coordinates shows

$$
\sigma\left(N_{\rho}^{r}\right)=\frac{n-1}{\pi} \int_{-\rho^{2} r}^{\rho^{2} r} \int_{1-\rho^{2} r^{2}}^{1}\left(1-t^{2}\right)^{n-2} t d t d \theta \sim \rho^{2 n} r^{2 n-1} .
$$

Hence we need only show that $c, c^{\prime}$ exist such that for $\rho^{2}=1-R^{2}$

$$
N_{\rho}^{c r} \subseteq Q_{R}^{r}\left(e_{1}\right) \subseteq N_{\rho}^{c^{\prime} r}
$$

Proof that $N_{\rho}^{c r} \subseteq Q_{R}^{r}\left(e_{1}\right)$ : We need to show that if $c$ is small enough, $\zeta \in S$ and $\zeta_{1}=t e^{i \theta}$ with $|\theta|<c\left(1-R^{2}\right) r$ and $0<1-t<c^{2}\left(1-R^{2}\right) r^{2}$, then there exists $R^{\prime}$ such that $R^{\prime} \zeta \in V_{r}\left(R e_{1}\right)$ (so that $\zeta \in \pi\left(V_{r}\left(R e_{1}\right)\right)$ ). In fact this holds with $R^{\prime}=R$ :

One checks that $R \zeta \in \phi_{R l_{1}}(r B)$ if and only if $\left|1-R^{2} \zeta_{1}\right|^{2}<\left(1-R^{2}\right)^{2} /\left(1-r^{2}\right)$. If $\zeta_{1}=t e^{i \theta}$ where $t, \theta$ are as above, then

$$
\begin{aligned}
\left|1-R^{2} \zeta_{1}\right|^{2} & =2 R^{2} t(1-\cos \theta)+\left(R^{2}(1-t)+1-R^{2}\right) \\
& <\theta^{2}+\left((1-t)+1-R^{2}\right)^{2}<\left(1+3 c^{2} r^{2}+c^{4} r^{4}\right)\left(1-R^{2}\right)^{2} .
\end{aligned}
$$

If $c$ is small enough, then $1+3 c^{2} r^{2}+c^{4} r^{4}<1 /\left(1-r^{2}\right)$ for $0 \leqslant r \leqslant \frac{1}{2}$. 
Proof that $Q_{R}^{r}\left(e_{1}\right) \subseteq N_{\rho}^{c^{\prime} r}$ : We need to show that if $z \in V_{r}\left(R e_{1}\right)$ and $z /|z|=\zeta$ $=\left(\zeta_{1}, \zeta^{\prime}\right)$ where $\zeta_{1}=t e^{i \theta}$, then $1-t<\left(c^{\prime}\right)^{2}\left(1-R^{2}\right) r^{2}$ and $|\theta|<c^{\prime}\left(1-R^{2}\right) r$. As on p. 29 of [RU], $z \in V_{r}\left(r e_{1}\right)$ implies

$$
\frac{\left|z_{1}-\alpha\right|^{2}}{r^{2} \beta^{2}}+\frac{\left|z^{\prime}\right|^{2}}{r^{2} \beta}<1
$$

where

$$
\alpha=\frac{\left(1-r^{2}\right) R}{1-r^{2} R^{2}}, \quad \beta=\frac{1-R^{2}}{1-r^{2} R^{2}} .
$$

It follows that $\left|z_{1}-R\right|<c_{1} r\left(1-R^{2}\right)$ (which implies $\left|z_{1}\right| \geqslant c_{2}>0$ so $|z| \geqslant c_{2}$ ) and that $\left|z^{\prime}\right|<c_{3} r\left(1-R^{2}\right)^{1 / 2}$. Hence

$$
1-t=1-\frac{\left|z_{1}\right|}{|z|} \leqslant 1-\frac{\left|z_{1}\right|^{2}}{|z|^{2}}=\frac{\left|z^{\prime}\right|^{2}}{|z|^{2}}<\left(c^{\prime}\right)^{2} r^{2}\left(1-R^{2}\right)
$$

and

$$
\begin{aligned}
|\theta| & \leqslant c_{4}\left|1-\frac{z_{1}}{\left|z_{1}\right|}\right| \leqslant c_{5}|| z_{1}\left|-z_{1}\right| \leqslant c_{5}\left(|| z_{1}|-R|+\left|z_{1}-R\right|\right) \\
& \leqslant 2 c_{5}\left|z_{1}-R\right| \leqslant c^{\prime} r\left(1-R^{2}\right) . \quad \text { Q.E.D. }
\end{aligned}
$$

3.15 Definition. For $0<r \leqslant \frac{1}{2}$ and $z \in B$, let

$$
f_{r}(z)= \begin{cases}1, & |z|<r \\ 0, & |z| \geqslant r\end{cases}
$$

For $\zeta \in S$, let $M_{r} \mu(\zeta)=\sup _{0 \leqslant R<1} \mu * f_{r}(R \zeta)$, where $\mu * f(z)=\int_{B} f \circ \phi_{z} d \mu$.

3.16 Lemma. For $0<r \leqslant \frac{1}{2}$ and $\lambda>0$

$$
\sigma\left(\left\{\zeta \in S: M_{r} \mu(\zeta)>\lambda\right\}\right) \leqslant \frac{c r^{2 n-1}}{\lambda} \int_{B} g d \mu .
$$

Proof. Define a measure $\mu_{0}$ in $B$ by $d \mu_{0}=g d \mu$. Fix $\lambda>0$; let $E=\{z \in B$ : $\left.\mu * f_{r}(z)>\lambda\right\}$. Note that for $z \in E$ we have $\mu\left(V_{r}(z)\right)>\lambda$ so that $\mu_{0}\left(V_{r}(z)\right)>$ $c\left(1-|z|^{2}\right)^{n} \lambda$. Using Lemma 3.13, a standard argument (see, e.g., [RU, Lemma 5.2.3]) allows as to find a family $\left\{z_{\alpha}\right\} \subseteq E$ such that $\left\{V_{r}\left(z_{\alpha}\right)\right\}$ are pairwise disjoint, but $E \subseteq \bigcup_{\alpha} V_{3 r}\left(z_{\alpha}\right)$. Let $\zeta_{\alpha}=z_{\alpha} /\left|z_{\alpha}\right|, R_{\alpha}=\left|z_{\alpha}\right|$ and $Q_{\alpha}=Q_{R_{\alpha}}^{3 r}\left(\zeta_{\alpha}\right)$; then $\{\zeta \in S$ : $\left.M_{r} \mu(\zeta)>\lambda\right\} \subseteq \cup_{\alpha} Q_{\alpha}$. Hence

$$
\begin{aligned}
\sigma\left(\left\{\zeta \in S: M_{r} \mu(\zeta)>\lambda\right\}\right) & \leqslant \sum_{\alpha} \sigma\left(Q_{\alpha}\right) \leqslant c r^{2 n-1} \sum_{\alpha}\left(1-R_{\alpha}^{2}\right)^{n} \\
& \leqslant \frac{c r^{2 n-1}}{\lambda} \sum_{\alpha} \mu_{0}\left(V_{r}\left(z_{\alpha}\right)\right) \\
& \leqslant \frac{c r^{2 n-1}}{\lambda}\left\|\mu_{0}\right\|=\frac{c r^{2 n-1}}{\lambda} \int_{B} g d \mu,
\end{aligned}
$$

since the $V_{r}\left(z_{\alpha}\right)$ are disjoint. Q.E.D. 
3.17 Definition. For $\zeta \in S$

3.18 LEMMA. If $r_{j}=2^{-j /(2 n-2)}$, then

$$
M^{\prime} \mu(\zeta)=\sup _{0 \leqslant R<1} \int_{B} G_{0}(R \zeta, w) d \mu(w) .
$$

$$
M^{\prime} \mu \leqslant c \sum_{j=0}^{\infty} 2^{j} M_{r_{j}} \mu .
$$

Note. We are assuming $n>1$ here!

Proof. This is immediate from the fact that $g_{0} \leqslant c \sum_{j=0}^{\infty} 2^{j} f_{r_{j}}$, which follows in turn from the fact that $g(z) \sim|z|^{-2 n+2}$ for $|z| \leqslant \frac{1}{2}$. Q.E.D.

3.19 Proposition. For $\lambda>0$

$$
\sigma\left(\left\{\zeta \in S: M^{\prime} \mu(\zeta)>\lambda\right\}\right) \leqslant \frac{c}{\lambda} \int_{B} g d \mu .
$$

Proof. Fix $\lambda>0$; let $E=\left\{\zeta \in S: M^{\prime} \mu(\zeta)>\lambda\right\}$. Pick $\alpha$ with $0<\alpha<$ $1 /(2 n-2)$. Lemma 3.18 shows that

$$
M^{\prime} \mu \leqslant c \sum_{j=0}^{\infty} 2^{-\alpha j} 2^{(1+\alpha) j} M_{r_{j}} \mu \leqslant c \sup _{j \geqslant 0} 2^{(1+\alpha) j} M_{r_{j}} \mu .
$$

Hence $E \subseteq \bigcup_{j=0}^{\infty} E_{j}$, where $E_{j}=\left\{\zeta \in S: M_{r_{j}} \mu(\zeta)>c 2^{-(1+\alpha) j} \lambda\right\}$. Thus Lemma 3.16 shows

$$
\begin{aligned}
\sigma(E) & \leqslant \sum_{j} \sigma\left(E_{j}\right) \leqslant c \int_{B} g d \mu \sum_{j} r_{j}^{2 n-1}\left(2^{-(1+\alpha) j} \lambda\right)^{-1} \\
& =\frac{c}{\lambda} \int_{B} g d \mu \sum_{j} 2^{-j(1 /(2 n-2)-\alpha)} \\
& =\frac{c}{\lambda} \int g d \mu . \quad \text { Q.E.D. }
\end{aligned}
$$

3.20 Definition. For $\zeta \in S$

$$
M \mu(\zeta)=\sup _{0 \leqslant R<1} \int_{B} G(R \zeta, w) d \mu(w) .
$$

Combining Corollary 3.8 and Proposition 3.19 establishes

3.21 TheOREM. For $\lambda>0$

$$
\sigma(\{\zeta \in S: M \mu(\zeta)>\lambda\}) \leqslant \frac{c}{\lambda} \int_{B} g d \mu .
$$

Since $\int_{B} G(z, w) d \mu(w) \rightarrow 0$ uniformly as $|z| \rightarrow 1$ if $\mu$ has compact support, Theorem 3.21 implies Theorem 3.3 by a standard argument: let $\varepsilon>0$. Let $\mu=\mu_{1}+$ $\mu_{2}$ where $\mu_{1}$ has compact support, $\int_{B} g d \mu_{2}<\varepsilon^{2} / c$ (this " $c$ " is as in the statement of Theorem 3.21). Then

$$
\begin{aligned}
\{\zeta \in S: & \left.\limsup _{R \rightarrow 1} \int_{B} G(R \zeta, w) d \mu(w)>\varepsilon\right\} \\
& =\left\{\zeta \in S: \limsup _{R \rightarrow 1} \int_{B} G(R \zeta, w) d \mu_{2}(w)>\varepsilon\right\} \\
& \subseteq\left\{\zeta \in S: M \mu_{2}(\zeta)>\varepsilon\right\} .
\end{aligned}
$$


So Theorem 3.21 shows that

$$
\sigma\left(\left\{\zeta \in S: \limsup _{R \rightarrow 1} \int_{B} G(R \zeta, w) d \mu(w)>\varepsilon\right\}\right)<\varepsilon
$$

for any $\varepsilon>0$; thus $\int_{B} G(R \zeta, w) d \mu(w) \rightarrow 0$ a.e. Q.E.D.

4. A counterexample. As mentioned in the introduction, Theorem 3.1 becomes false if radial limits are replaced by limits in any sort of "approach region": Let $\Omega$ be any open subset of $B$ containing $\left\{r e_{1}: 0 \leqslant r<1\right\}$. Shrinking $\Omega$ if necessary, assume that $U \Omega=\Omega$ for all $U \in \mathscr{U}$ such that $U e_{1}=e_{1}$ (i.e., if $\left(z_{1}, z^{\prime}\right) \in \Omega$ and $\left|w^{\prime}\right|=\left|z^{\prime}\right|$, then $\left(z_{1}, w^{\prime}\right) \in \Omega$ ). For $\zeta \in S$, set $\Omega_{\zeta}=U \Omega$, where $U \in \mathscr{U}$ is such that $U e_{1}=\zeta$. (Our hypothesis on $\Omega$ implies that $\Omega_{\zeta}$ is well defined.)

4.1 EXAMPLE. There exists an $M$-sh function $u \leqslant 0$ in $B$ such that for almost all $\zeta \in S$

$$
\liminf _{z \rightarrow \zeta, z \in \Omega_{\zeta}} u(z)=-\infty
$$

while

$$
\limsup _{z \rightarrow \zeta, z \in \Omega_{\zeta}} u(z)=0 .
$$

Proof. Let $\left\{z_{j}\right\}$ be a discrete subset of $B$ such that every $\Omega_{\zeta}$ contains infinitely many $z_{j}$ 's. Let $c_{j}>0$ be such that $\sum c_{j} g\left(z_{j}\right)<\infty$. Let $\mu=\sum c_{j} \delta_{j}$, where $\delta_{j}$ is the point mass at $z_{j}$, and let

$$
u(z)=-\int_{B} G(z, w) d \mu(w) .
$$

Theorem 3.3 shows $u$ has radial limit 0 almost everywhere; on the other hand $u\left(z_{j}\right)=-\infty$ for all $j$. Q.E.D.

\section{REFERENCES}

[CS] J. A. Cima and C. S. Stanton, Admissible limits of M-subharmonic functions, Michigan Math. J. (to appear).

[HE] L. L. Helms, Introduction to potential theory, Wiley, New York, 1969.

[LW] J. E. Littlewood, On functions subharmonic in a circle. III, Proc. London Math. Soc. (2) 32 (1931), 222-234.

[RU] W. Rudin, Function theory in the unit ball of $\mathbf{C}^{n}$, Springer-Verlag, New York, 1980.

Department of Mathematics, OKlahoma State University, Stillwater, Oklahoma 74078 